\title{
Politique
}

\section{La réforme institutionnelle de la fonction publique de 1983 : contexte, contenu et enjeux}

James Iain Gow

Numéro 6, automne 1984

La réforme des institutions politiques

URI : https://id.erudit.org/iderudit/040461ar

DOI : https://doi.org/10.7202/040461ar

Aller au sommaire du numéro

Éditeur(s)

Société québécoise de science politique

ISSN

0711-608X (imprimé)

1918-6584 (numérique)

Découvrir la revue

Citer cet article

Gow, J. I. (1984). La réforme institutionnelle de la fonction publique de 1983 : contexte, contenu et enjeux. Politique, (6), 51-101.

https://doi.org/10.7202/040461ar d'utilisation que vous pouvez consulter en ligne.

https://apropos.erudit.org/fr/usagers/politique-dutilisation/ 


\title{
La réforme institutionnelle de la fonction publique de 1983:
}

\author{
contexte, contenu et enjeux
}

\author{
James Iain Gow \\ Université de Montréal
}

Le 21 décembre 1983 l'Assemblée nationale du Québec adopta une nouvelle Loi sur la fonction publique. Celle-ci vise à alléger le contrôle central exercé sur les gestionnaires afin de permettre à ceux-ci d'assumer plus de responsabilités pour la qualité des services offerts aux citoyens ainsi que pour l'économie et l'efficacité de leurs services. Le ministère de la Fonction publique est aboli par la loi, ses fonctions étant assumées par le Conseil du trésor ou bien par l'Office des ressources humaines (qui succède, lui, à l'Office du recrutement et de la sélection du personnel de la fonction publique). C'est une vraie réforme qui est ainsi effectuée, mais on ne saura la qualifier d'importante ou de mineure avant de connaître comment elle s'insérera dans la série de changements qu'elle annonce. Après avoir esquissé notre problématique, nous traiterons du contexte de la réforme, de son déroulement ainsi que des points de vue des différents intéressés. Notre bilan sera forcément provisoire: seul l'avenir nous dira si un changement important a été amorcé par la nouvelle loi ou si, au contraire, il ne s'agit que d'une réforme tronquée dans le genre de celle de 1978 ayant comme conséquence, la nécessité d'une nouvelle loi dans peu d'années. 


\section{Problématique}

Avant de proposer quelques hypothèses, nous devons préciser notre conception de la fonction publique et de la réforme administrative.

\section{La fonction publique comme institution}

Selon le Petit Robert les institutions sont: «L'ensemble des formes ou structures sociales telles qu'elles sont établies par la loi ou la coutume et spécialement celles qui relèvent du droit public. » Appliquée à la fonction publique, cette définition pourrait donner lieu à une interprétation étroite ou extensive. Selon une définition étroite, il serait question des structures de gestion du personnel qu'elles soient des organismes centraux ou les services de personnel des ministères et, peut-être, la structure des emplois, c'est-à-dire le système de classification. En fait, la plupart des définitions en administration publique donnent une interprétation plus large au mot «institution». Même les juristes, qui tendent vers une approche formelle vont inclure dans le domaine des institutions les règles de fonctionnement ainsi que la chose instituée, c'est-à-dire, l'ensemble des personnes, du matériel, des biens etc. qui la composent ${ }^{1}$. Ainsi, la fonction publique est elle-même une institution au même titre que les structures formelles qui l'instituent et la régissent ${ }^{2}$.

La sociologie des organisations amène une version plus large de la notion d'institution. L'école du comportement, à l'instar de Herbert Simon, considère qu'une organisation est un groupe de personnes poursuivant un ou plusieurs objectifs communs.

1. Cf. Henry Puget, Les institutions administratives à l'étranger, Paris, Dalloz, 1969; R. Ducos-Ader, Institutions administratives, Paris, Dalloz, 1966, 11.

2. Public Administration Revieu, "Symposium on the Public Service as Institution", vol. 42, 4 (juillet-août 1982), 304-320. 
Selon cette perspective, il n'y a pas de raison de postuler une distinction nette entre les structures et les autres formes d'influence sur le comportement des membres ${ }^{3}$. Les structures formelles définissent objectifs et rôles, tâches et responsabilités; elles sont complétées par des règlements, des politiques, des directives, mais aussi par les éléments informels que sont les coutumes et les habitudes. Ce sont les "règles du jeu» qui posent des limites aux stratégies que peuvent adopter les différents participants à l'organisation ${ }^{4}$.

Une approche sociologique considère qu'une organisation ne devient une institution que lorsqu'elle réussit à se donner des racines dans son milieu. Délaissant les questions de capacité de production et de règles de fonctionnement, elle en privilégie d'autres qui traitent de besoins, de pressions et de légitimité:

Appréhender l'administration comme appareil institutionnel, c'est privilégier l'étude des interrelations qui l'unissent au reste de la société et chercher à comprendre la logique sociale qui sous-tend son actions.

Il y a moyen d'intégrer ces différentes approches (juridique et sociologique, limitée ou extensive) par le recours à la notion du politique telle que développée par Vincent Lemieux. Celui-ci considère que «le politique consiste dans les relations de pouvoir par lesquelles se fait la gouverne des organisations ${ }^{6}$. En tant que systèmes finalisés d'action, les organisations doivent assurer la régulation des rapports entre leurs composantes ainsi que ceux qui les engagent vis-à-vis des intervenants dans leur environnement. Les règles formelles d'une institution visent précisément ces deux

3. Herbert Simon, Administrative Behavior, $2^{\circ}$ éd., New York, The Free Press, $1957,100-103$ et $147-149$.

4. Michel Crozier et Erhard Friedberg, L'acteur et le systeme, Paris, Le Seuil, 1977, $212-214$.

5. Michel Chevallier et Danièle Loschak, Science administrative, vol. 1, Paris, LGDJ, 1979, 175.

6. Vincent Lemieux, Les cheminements de l'influence, Québec, PUL, 1979, 5. 
types de relation, car non seulement assurent-elles la distribution des tâches et des compétences mais elles précisent qui peut engager l'organisation sur le plan extérieur et dans quelles circonstances.

Nous optons, donc, pour une définition extensive de l'institution de la fonction publique, définition qui comprend les règles de sa gouverne ainsi que la chose instituée, l'ensemble des personnes qui la composent à un moment donné. Essentiellement, il s'agit du groupe de fonctionnaires de carrière directement à l'emploi de l'État dans ses ministères et organismes. Nous essayerons de l'envisager aussi dans ses règles de fonctionnement interne et dans ses rapports avec les intervenants les plus importants dans son milieu à savoir, les ministres du gouvernement, les députés de l'Assemblée nationale et les syndicats.

\section{La réforme administrative}

Quant à la notion de réforme, elle évoque l'idée du changement planifiée ${ }^{7}$.

Action volontaire et concertée, la réforme administrative n'est jamais le fait du hasard, mais le résultat d'un processus dont on peut déceler les origines et les diverses étapes jusqu'au moment où il est fait un sort à la réforme.

À l'encontre des cas bien plus nombreux et probablement plus importants où l'administration se contente de s'adapter aux changements survenus dans son environnement, il s'agit de cas

7. Gérard Caiden, "Administrative reform» dans La revue internationale des sciences administratives, 1968, $347 \mathrm{~s}$, , repris dans Yves Chapel, Stratégies et tactiques de la modernisation publique dans les pays en développement (recueil de textes), Bruxelles, Institut international des sciences administratives, 1972, 18. Voir aussi du même auteur, "Administrative reform: a Prospectus", Revue internationale des sciences administratives (RISA), vol. 44, nos 1-2 (1978), 106-120 et Kenneth Cabatoff, "La réforme institutionnelle de la fonction publique » dans J. Bauer et K. Cabatoff (sld), Bilan de la loi 50; dynamique du changement de la fonction publique québécoise, Actes du premier colloque sur la réforme et le changement en administration publique, Université du Québec à Montréal, les 20-21 mars $1980,37-59$. 
de génie social ou de "design" organisationnel, où un ou des réformateurs essaient délibérément de changer le cours de l'évolution institutionnelle. Une telle défnition implique l'identification des participants à un processus de réforme ainsi que des valeurs privilégiées par ces acteurs. Activité consciente finalisée, la réforme administrative se prête bien au genre d'analyse stratégique que proposent Michel Crozier et Vincent Lemieux ${ }^{8}$.

Une bonne partie de la science administrative s'adresse au problème de la réforme administrative, depuis l'organisation scientifique du travail jusqu'aux méthodes les plus récentes de prise de décision et d'analyse budgétaire. Selon l'approche théorique que l'on adopte, le processus de réforme recommandé mettra l'accent sur des facteurs internes ou externes de l'organisation. La tendance de la science administrative normative est de privilégier les facteurs internes; par exemple Butani écrit qu'une réforme administrative ne pourra réussir que si l'urgence de la réforme est comprise par l'organisation, si ceux qui devront l'appliquer sont associés à sa conception, que si un organe ad boc est créé pour assurer le suivi et l'évaluation de la réforme et que si le personnel qui dirigera la réforme est choisi et formé soigneusement ${ }^{9}$. Ce genre d'idée inspire l'école du développement organisationnel selon laquelle il faut dégeler les attitudes des membres d'une organisation afin de les rendre réceptifs à la réforme ${ }^{10}$. Par contre l'approche des sociologues préoccupés par l'adéquation des structures aux exigences de leur environnement dirige notre attention sur

\footnotetext{
8. Crozier et Friedberg, op. cit., et Vincent Lemieux, op. cit., ch. 5 .

9. K. N. Butani, "Implementing Administrative Innovations and Reforms", The Indian Journal of Public Administration, July-Sept. 1966, teproduit dans Y. Chapel, op. cit., 39-42.

10. D.E. Zand et R.E. Sorenson, "Theory of Change and Effective use of Management Science", Administrative Science Quarterly, vol. 20 (1975), 532-545, Laurent Bélanger, "Les stratégies du développement organisationnel», Relations industrielles, vol. 27, 4 (1972), 633-651 et Public Administration Review, Symposium on Organizational Development", vol. 34, 2 (March-April 1974).
} 
le leadership, le choix de la technologie appropriée ${ }^{11}$ et le maintien de liens avec les groupes dans les milieux dont l'appui est essentiel pour obtenir les ressources nécessaires à la survie de l'institution ${ }^{12}$.

Deux autres remarques sont suggérées par la théorie. Règle générale, la réforme administrative s'inspire de valeurs économiques (l'efficience, l'efficacité et l'économie) ou bien politiques (l'égalité, la représentativité, l'impartialité et la liberté) ${ }^{13}$. Bon nombre de réformes essaieront de promouvoir les deux types de valeur en même temps, mais on conçoit facilement qu'elles puissent être en contradiction les unes avec les autres. Finalement, la réforme administrative aura des conséquences attendues par ses auteurs, mais elle en aura d'autres qui ne seront ni attendues ni voulues ${ }^{14}$.

Nous pouvons maintenant résumer notre pensée sur la réforme administrative et l'appliquer à la réforme actuelle de la fonction publique québécoise. Pour réussir, une réforme doit s'inspirer d'une volonté de changement, elle doit trouver un catalyseur pour l'initier et la poursuivre, elle doit obtenir la collaboration d'une bonne partie sinon de tous les fonctionnaires et elle doit ou bien avoir des appuis dans les milieux environnant ou bien apporter une adaptation aux conditions qui prévalent dans ce milieu.

Dans le cas présent, la volonté de réforme a trois sources principales. En premier lieu, après une période où le gouvernement du Parti québécois avait comme objectif principal de maîtriser la fonction publique afin de pouvoir mettre en vigueur son programme, la crise financière des dernières années lui a inspiré surtout le

11. Chevallier et Loschak, op. cit., 238.

12. Philip Selznick, Leadership in Administration et Milton Esman, "The Elements of Institution Building» dans Eaton (ed.), Institution Building and Development, Beverly Hills, Sage, 1972. C'est aussi la base de la "contigency theory qu'on doit à J.W. Lorsch et P.R. Lawrence, Organization and Environment, Homewood, Ill., Irwin, 1969.

13. Herbert Kaufman, "Emerging Conflicts in the Doctrines of Public Administration", American Political Science Review, vol. 5 (déc. 1956), 1057-1073, cité par K. Cabatoff, op. cit., 41.

14. Robert King Merton, Éléments de la méthode sociologique, Paris, Plon, 118. 
désir de trouver les moyens de maintenir la qualité des services tout en coupant dans les dépenses. En second lieu, il y a un mouvement grandissant d'impatience du côté des députés face au monopole d'initiative gouvernementale et à l'autonomie grandissante de l'administration. Troisièmement, il y a la déception des principaux intéressés, de la ministre mais surtout des hauts fonctionnaires, à propos des résultats de la réforme des années 1977-1978 qui aboutit au projet de loi 50.

Si le gouvernement contrôle l'ensemble du processus, le catalyseur est la Commission spéciale de l'Assemblée nationale sur la fonction publique, mieux connue par le nom de son président, Guy Bisaillon. Créée en novembre 1981, la Commission Bisaillon consultera largement les membres de la fonction publique ainsi que les groupes et institutions externes avant de faire son rapport en juin 1982. La ministre de la Fonction publique deviendra la marraine de l'Avant projet de loi déposé à l'Assemblée nationale en juin 1983, puis du projet de loi 51 qui sera adopté en décembre 1983.

Nous passons maintenant au contexte de la réforme, afin d'identifier les forces tendant vers un changement. Ensuite, il sera question du processus, depuis son déclenchement par le catalyseur jusqu'à son aboutissement avec l'adoption du projet de loi 51 . Notre analyse du contenu de ce projet ainsi que des prises de position des différents intervenants dans le débat révélera les enjeux pour ceux qui sont de l'administration et pour ceux de l'extérieur qui attendent quelque chose d'elle.

\section{Les forces favorisant le changement}

Il s'agit, dans cette deuxième partie, d'examiner les problématiques des groupes ou acteurs intéressés par la réforme de la fonction publique. La position du gouvernement nous retient le plus longuement, car le projet de loi 51 est sa deuxième réforme 
en lã matière en six ans au pouvoir. Il sera question ensuite de la position des parlementaires avant de passer à des considérations plus techniques qui inspirent les hauts fonctionnaires face à la révision de la loi de 1978.

\section{L'évolution de la position gouvernementale}

Pour bien comprendre la position du gouvernement péquiste lors de la réforme de 1983, il faut remonter au lendemain de l'élection de 1976, car ses attitudes évoluent avec le temps.

Sa position face à l'administration connaît trois périodes qui se chevauchent mais qui sont quand même identifiables. Au début de son mandat, le gouvernement cherche surtout à s'assurer le contrôle afin de réaliser son programme sans dilution, distortion ou retardement à cause des positions de force des fonctionnaires. Ensuite, en 1979-80, avec les négociations du secteur public et le référendum, il est plus sensible au poids politique des fonctionnaires. Dès cette époque, mais surtout à partir de 1981, il tient le discours de la crise financière et invite la fonction publique, comme le secteur public d'ailleurs, à faire plus avec moins.

\section{6-1979: prendre le pouvoir}

Au début du premier mandat du gouvernement du Parti québécois, la fonction publique avait toutes les raisons de croire qu'elle connaîtrait de meilleurs jours.

À la fin du mandat du gouvernement libéral de Robert Bourassa, son moral était très bas à la suite de scandales, de régimes d'exception et de nombreux cas de patronage ${ }^{15}$. Or, les dirigeants du Parti québécois avaient pris la défense des fonc-

15. Cf. notre texte "Le contexte historique de la réforme administrative» dans Bauer et Cabatoff, Bilan de la loi 50, 60-73. 
tionnaires à ce moment-là et ils avaient fait campagne en promettant d'être un «bon gouvernement». Leur programme d'inspiration social-démocrate laissait croire à un rôle accru des fonctionnaires du fait des interventions étatiques nombreuses qu'il impliquait. Finalement, la présence au gouvernement d'anciens conseillers ou fonctionnaires comme Jacques Parizeau, Claude Morin et Denis de Belleval pouvait faire croire aux fonctionnaires qu'ils seraient compris par leurs nouveaux maîtres ${ }^{16}$. Peut-être étaient-ils trop bien compris, car le gouvernement a pris les moyens pour éviter toute dépendance vis-à-vis de la haute fonction publique.

Ces moyens prenaient diverses formes. En introduisant un nouveau type de poste, celui de ministre d'État, le gouvernement s'est donné une capacité d'analyse indépendante des ministères. Les ministres d'État étaient tous des personnalités fortes bien en vue au sein du Parti québécois ${ }^{17}$. Pour les seconder dans leurs tâches de présidents des comités sectoriels du Conseil des ministres, ils avaient des effectifs restreints (de dix à douze personnes chacun) choisis librement sans concours, car ils avaient le statut soit de sous-ministre, soit de membre de cabinet ministériel ${ }^{18}$. En plus

16. Philippe Gagnon, "L'euphorie s'empare de la fonction publique», La Presse du 24 novembre 1976 et Alain Baccigalupo, «Le PQ au pouvoir laisse espérer la réalisation prochaine d'une vaste réforme administrative" dans D. Latouche et al., Premier mandat et repris dans son livre Les grands rouages de la machine administrative québécoise, Montréal, Éditions Agence d'Arc, 1978, 569-589.

17. Ce système est décrit par Louis Bernard dans «Les mécanismes centraux de coordination au Québec», au Colloque international de l'ENAP sur l'Administration publique: perspectives d'avenir, Québec, le $30 \mathrm{mai}, 1979$ et dans les rapports annuels du ministère du Conseil exécutif pour 1978-1979 et 1979-1980. Voir aussi Lysiane Gagnon, "La structure du pouvoir dans le cabinet Lévesque: les "cardinaux" et les "évêques»: un équilibre délicat", La Presse du 16 novembre 1978 et Pierre O'Neill er Jacques Benjamin, Les mandarins du pouvoir, Montréal, Québec/Amérique, 1978, 218 224.

18. Jacques Champagne dans une communication sur les «Structures of Policy Planning in Quebec» à la réunion de l'Association canadienne de science politique à Saskatoon, 1979, estime que la moitié des effectifs des ministères d'État provient des fonctions publiques québécoise et canadienne et l'autre moitié du secteur privé. Voir aussi O'Neill et Benjamin, op. cit., 225-226. 
de renforcer sa capacité d'initiative, d'étude et de coordination au sommet de l'appareil, le gouvernement prend d'autres moyens afin d'assurer son emprise sur l'administration. D'une part, les membres des cabinets ministériels sont plus nombreux et plus instruits que leurs prédécesseurs et ils participent davantage non seulement à la prise de décision mais aussi à la gestion courante de leur ministère ${ }^{19}$. D'autre part, si le Premier ministre ne sabre pas parmi les effectifs des sous-ministres hérités du gouvernement précédent, au cours des premiers dix-huit mois, plus de la moitié sont mutés ou partent de leur propre gré ${ }^{20}$. Plus important encore, on se sert du poste de sous-ministre adjoint pour placer un bon nombre de personnes partisanes du Parti québécois dans les cadres supérieurs de l'administration ${ }^{21}$.

Donc, au début de 1979, les rapports entre le politique et l'administratif ont passablement changé au Québec. La direction politique est renforcée et le sommet de l'appareil administratif est davantage politisé. Outre ces nominations, la politisation est évidente au ministère du Conseil exécutif (donc, du Premier ministre). Depuis la création du poste de Secrétaire général en 1968 , ce poste, comme celui de Greffier auparavant, avait été comblé par un homme de l'administration qui jouissait de la confiance du Premier ministre, mais qui n'était pas considéré comme un attaché partisan comme l'était, par exemple, le chef de cabinet. Or, avec la nomination en avril 1978 de Louis Bernard, cette

19. Micheline Plasse, «Les chefs de cabinets ministériels au Québec: la transition du gouvernement libéral au gouvernement péquiste (1976-1977) dans la Revue canadienne de science politique, vol. 14, 2 (juin 1981) notamment aux pages 329-335 et O'Neill et Benjamin, op. cit., 225-228.

20. Selon le Répertoire des employés supérieurs (bors cadre) des ministères du gouvernement du Québec 1867-1983, préparé pour la Bibliothèque de l'Assemblée nationale, par Jacques Bourgault, Québec, 1983, 12 sous-ministres sur 22 en ce 15 novembre 1976 ne sont plus là après un an. Voir aussi Lysiane Gagnon, "Ce qui est bon pour le PQ...», La Presse du 20 janvier 1979. 225-228.

21. Lysiane Gagnon, "Ce qui est bon pour le PQ ...» et O'Neill et Benjamin, 
tradition est rompue. M. Bernard est un militant du Parti québécois qui continue à s'identifier comme tel une fois nommé à ce poste au sommet de la pyramide administrative. Il développe, au cours d'entrevues et de conférences, la pensée du gouvernement sur cette question. Il est normal dit-il que le personnel politique désire prendre le dessus après une période de domination technocratique. Les changements introduits par le gouvernement visent à lui permettre de choisir en fonction d'analyses aussi bien politique que technique. Dans ce processus le fonctionnaire retrouve son rôle normal d'analyste technique et d'exécutant; il est au politicien ce que l'ingénieur est à l'architecte ${ }^{22}$. Ceci a un effet démoralisant sur la haute fonction publique qui se sent écartée de plusieurs grandes décisions et les occasions de conflits ne manquent pas entre les cadres supérieurs et les membres (plus jeunes et plus partisans) des cabinets ministériels ${ }^{23}$.

En ce qui concerne les autres fonctionnaires, le gouvernement fait amender la Loi de la fonction publique par le projet de loi 50 adopté en juin 1978. En préparant son projet original (la loi 53 de 1977), le gouvernement, contrairement à sa pratique dans presque tous les secteurs de la vie sociale et économique, a évité de consulter les syndicats de fonctionnaires jusqu'au moment du dépôt du projet de loi. Leur résistance fut si grande que le gouvernement dut retirer son projet et revenir avec une nouvelle version à la session suivante. Nous reviendrons sur cette loi plus loin; pour le moment on retiendra la stratégie gouvernementale

22. Jean-Claude Picard, «Louis Bernard: le fonctionnaire est au politicien ce que l'ingénieur est à l'architecte", Le Devoir du 14 septembre 1978, et idem, "La fonction publique devra se décentraliser au profit des pouvoirs locaux et régionaux", Le Devoir du 21 janvier 1979.

23. Guy Rocher, "Le sociologue et la sociologie dans l'administration publique et l'exercice du pouvoir politique», Sociologie et sociétés, vol. 11, 2, (oct. 1980), 51, M. Plasse, loc. cit., 332 et Roger Brissette, La conception des bauts fonctionnaires québécois face aux cabinets ministériels, mémoire de maîtrise (science politique), Université de Montréal, 1981. 
face aux fonctionnaires de 1976 à 1979. Il s'agissait, selon un mot attribué à René Lévesque à l'époque, après avoir gagné les élections de gagner le pouvoir ${ }^{24}$.

\section{9-1980: sortir indemne des négociations et gagner le référendum}

Les années 1979-1980 sont une période de confrontation politique pour le gouvernement péquiste, d'abord, lors des négociations avec les syndicats du front commun syndical et d'autres de la fonction publique et du secteur public, ensuite, lors de la campagne référendaire de 1980 . Il semble que le 'gouvernement ait été plus sensible au poids politique des employés publics à ce moment que par la suite.

À l'été de 1979, le gouvernement se trouve en négociations collectives avec les représentants de 275000 employés des secteurs publics et parapublics, dont 200000 membres du Front commun (surtout des réseaux de l'éducation et des affaires sociales) ${ }^{25}$. Les syndicats des fonctionnaires professionnels (le SPGQ) et des enseignants de l'État (SPEQ) négocient séparément au nom de leurs 7000 membres, comme le fait déjà depuis un an le syndicat des fonctionnaires provinciaux (SFPQ) pour le compte de ses 26500 membres.

L'attitude du gouvernement face aux syndicats du secteur public semble ambivalente. En principe, il leur est favorable. Au printemps de 1977, il a laissé tomber plus de 7000 poursuites intentées par le gouvernement Bourassa à la suite des négociations de 1975-1976. Puis, au moment de mettre sur pied un nouveau régime de négociations, en 1978, il adopte une méthode inusitée

24. O'Neill et Benjamin, op. cit., 217.

25. Conseil d'information sur les négociations dans les secteurs public et parapublic, Rapport-synthèse sur l'état des négociations au 30 juin 1979, sl. sd. 
pour régler l'épineuse question des services essentiels à maintenir en cas de grève dans les établissements des affaires sociales: à défaut d'une entente entre les parties patronale et syndicale, la liste proposée par le syndicat prévaudra. Le gouvernement pourra suspendre le droit de grève pendant trente jours dans un établissement, s'il est persuadé qu'une grève met en danger la santé ou la sécurité du public ${ }^{26}$.

À l'épreuve, les nouveaux mécanismes ne satisfont pas le gouvernement. Après quelques arrêts de travail dans la fonction publique et dans plusieurs hôpitaux, et face à la possibilité de grèves plus étendues dans les secteurs de l'éducation et des affaires sociales, le gouvernement adopte une loi d'urgence le 12 novembre 1979 qui suspend le droit de grève dans tout le secteur public jusqu'au 30 novembre, afin de lui permettre de déposer de nouvelles offres. Cette tactique lui permettra de conclure des ententes avec le SFPQ et les syndicats des affaires sociales. Au mois de décembre, il utilise une seconde loi spéciale pour mettre fin, cette fois-ci, à une grève à l'Hydro-Québec. Il devra subir une grève des enseignants à la fin de janvier et au début de février 1980 avant d'arriver à une entente le 11 février. Le Syndicat des professionnels persiste dans son opposition jusqu'à la veille du référendum, le 16 mai.

Or, malgré le constat d'échec des mécanismes nouveaux d'encadrement des négociations, celles-ci se sont déroulées de manière relativement calme et sans heurt majeur ${ }^{27}$. Néanmoins, le gouvernement paraît incommodé par les grèves et autres incidents. L'opposition attrribue l'adoption de la loi spéciale au fait qu'il y a trois élections partielles le 14 novembre. Le lendemain de la défaite des candidats péquistes dans les trois comtés, plusieurs

26. Art. 4 du projet de loi 59 amendant l'article 99 du Code du travail, sanctionné le 23 juin 1978.

27. Conseil sur le maintien des services de santé et des services sociaux Rapportsyntbèse, juin 1980, 9-14, et François Demers, Chroniques impertinentes du 3'Front commun, Montréal, Nouvelle optique, 1982, 139. 
députés l'attribuent aux demandes qu'ils jugent exagérées des dirigeants syndicaux ${ }^{28}$.

Certes, les syndicats du secteur public ont fait des gains importants: 1) le salaire minimum de $265 \$$ est accordé pour la dernière année du contrat; 2) un congé de maternité de 20 semaines ${ }^{29}$; 3) une clause de sécurité d'emploi qui permet à l'employé de refuser toute réaffectation en cas de manque de travail au-delà de $50 \mathrm{~km}$ de son lieu de travail; et 4) pour les enseignants, un nombre maximum d'élèves par classe. Les fonctionnaires n'ont pas obtenu tous ces avantages, en particulier, ils n'ont pas eu la sécurité d'emploi régionale (la clause de $50 \mathrm{~km}$ ). Le SFPQ se dit néanmoins content, car sa convention apporte des gains aux membres sur les plans salarial, de carrière et de griefs. Quant aux professionnels, leur résistance leur apporte quelques gains: leur semaine de travail reste à $32 \frac{1}{2}$ heures au lieu d'être augmenté à 35 heures comme le voulait la demande patronale, et ils obtiennent une sorte de sécurité d'emploi régionale d'une durée de 18 mois après laquelle ils devront accepter toute autre réaffectation qui leur est proposée.

Les concessions gouvernementales ont toutes été accordées avant la loi spéciale du 12 novembre. Cette générosité était due, aux yeux des syndiqués, à l'approche du référendum de mai $1980^{30}$. Certes, le référendum était très présent dans l'esprit de ceux qui commentaient les résultats des trois élections partielles du 14 novembre. Aussi, pendant la campagne référendaire, Pierre Bourgault a dénoncé ce qu'il appelait le «chantage» pratiqué par les fonctionnaires professionnels qui essayaient d'obtenir gain de cause en menaçant de voter non dans le cas contraire ${ }^{31}$. En tout cas,

28. La Presse du 16 novembre 1979.

29. Selon Lise Payette dans Le pouvoir? connais pas!, Montréal, Québec/Amérique 1982, 55, c'est «un des congés de maternité les plus généreux en Amérique du Nord».

30. F. Demers, $o p$. cit., 131-136.

31. Le Devoir du 8 mars 1980. 
l'approche du référendum met en relief l'importance des fonctionnaires en tant que citoyen. Le 13 mars le ministre de la fonction publique, François Gendron, publie une directive indiquant que les fonctionnaires québécois pourront participer à la campagne référendaire, la raison étant que la Loi de la fonction publique n'interdit que des activités partisanes pendant une campagne électorale ${ }^{32}$.

L'attention des deux camps lors du référendum porte d'abord et surtout sur les 130000 fonctionnaires fédéraux demeurant au Québec. Fidèle en cela à un discours qu'il tient depuis longtemps, le gouvernement promet d'intégrer ces personnes à la fonction publique ou au secteur public d'un Québec souverain et ce, sans perte de salaire ${ }^{33}$. Loin de susciter des craintes chez les fonctionnaires du Québec, cette politique semble les encourager. D'après une annonce publiée dans Le Devoir le 3 avril 1980 par un "Comité fonctionnaires Québec Oui», la souveraineté-association impliquerait pour eux la parité de salaire avec les fonctionnaires fédéraux, donc une hausse de 10 à $15 \%$ et des chances d'avancement doublées.

Il est impossible de savoir si de telles considérations ont beaucoup influencé les fonctionnaires lors du vote référendaire. De par leur niveau d'instruction, leur type d'emploi et le degré élevé de syndicalisation, ils appartenaient de toute façon au groupe le plus apte à favoriser la souveraineté-association. Par contre, même si le vote était très partagé dans la région de la capitale, seul un comté sur cinq de la Ville de Québec a voté oui au référendum.

\section{1-1983: gérer la crise}

Même si le souci d'économie était présent dans son discours depuis son élection en 1976, on peut situer le recours à un langage de

32. Ibid., du 14 mars 1980.

33. Ibid., du 25 mars 1980 et The Gazette du 11 septembre 1972. 
crise financière à l'été de 1981. Dans son discours sur le budget du 10 mars 1981, le ministre Parizeau prévoit une période difficile en raison de la situation économique pénible, les taux d'intérêts très élevés et l'intention du gouvernement fédéral de réduire ses paiements de transfert aux provinces. Il annonce des «compressions budgétaires»; celles-ci ne sont pas des coupures, elles représentent des réductions dans les coûts qu'il aurait fallu payer simplement pour maintenir les services existants, compte tenu des taux prévus de salaires, d'inflation et d'intérêt ${ }^{34}$. Ainsi, limiter les hausses de dépenses en 1981-1982 à 13\%, représente úne «économie»de $5 \%$ par rapport à la hausse prévisible et, en même temps, un resserrement considérable. Dans la fonction publique les effectifs sont plafonnés, et dans douze ministères sur vingt-quatre les hausses de dépenses prévues de moins de $5 \%$ sont bien inférieures au taux d'inflation de $12 \%$. C'est dans les secteurs de l'éducation et des affaires sociales que les coupures les plus importantes sont annoncées: $\$ 500$ millions. Néanmoins, le ministre annonce une nouvelle réduction des impôts et une augmentation des exemptions personnelles.

$\mathrm{Au}$ lendemain de l'élection du 14 avril qui le reporte au pouvoir avec une marge de sièges de presque deux contre un, le Premier ministre décide de confier le poste de président du Conseil du trésor à Yves Bérubé, tout en maintenant Jacques Parizeau aux Finances. Le fait de séparer ces deux postes pour la première fois souligne le désir du Premier ministre d'accorder une attention particulière au contrôle des dépenses car, désormais il y aura un ministre pour les revenus et un autre pour les dépenses. À l'été dé 1981 M. Bérubé entreprend une campagne d'information pour sensibiliser le public à la nécessité des compressions budgétaires.

La problématique gouvernementale identifie les compressions des dépenses comme moyen principal d'un redressement financier,

34. Discours sur le budget du 10 mars 1981, 27-28. 
car les autres semblent tous bloqués: on ne peut plus financer les dépenses courantes par des déficits, car la dette publique augmente à un rythme alarmant, les Québécois paient déjà des taxes plus élevées que les citoyens des autres provinces, et les taux d'inflation et d'intérêt échappent à son contrôle. Reste alors les dépenses et l'objectif déclaré du gouvernement devient une réduction des dépenses réelles sans baisse des services rendus à la population. Ceci pourra se faire pendant un temps en cherchant à éliminer "le gras» qu'on croît déceler un peu partout dans le secteur public. De plus en plus, le discours gouvernemental sera axé sur le besoin d'accroître la productivité et de restreindre les salaires des employés du secteur public ${ }^{35}$.

Il faut dire que c'est un thème présent depuis longtemps dans le discours du Parti québécois. Dans son programme de 1975, au chapitre traitant de l'administration on énonce le principe suivant:

Les normes d'efficacité et de rentabilité appliquées dans l'entreprise privée prévalent dans l'administration publique. Après un an au pouvoir, dans son discours inaugural du 21 février, René Lévesque dit, "collectivement c'est très simple, nous ne travaillons pas assez, nous Québécois» et il vise particulièrement les employés des secteurs public et parapublic qui sont devenus "à la fois la locomotive et le wagon de toute la société " ${ }^{36}$.

À l'automne de 1981 et au printemps de 1982, avec l'échéance des conventions collectives à l'horizon, ce thème est de plus en plus mis en relief. Les fonctionnaires et autres employés publics sont devenus des personnes «extraordinairement privilégiées»_compte

35. Bernard Descoteaux, "Yves Bérubé: le Québec devra traverser encore deux années d'austérité", Le Devoir du 30 juillet 1981 . Voir aussi Les compressions budgétaires dans les secteurs de l'éducation et des affaires sociales. Rapport des délibérations de la rencontre publique tenue à Montréal le 12 juin 1981 dans le cadre des conférences socio-économiques, Québec, 1981, 18-27.

36. Le Devoir du 22 février 1978. 
tenu de leur rémunération et de leur sécurité d'emploi en période de récession ${ }^{37}$. À la conférence au sommet économique qui réunit des représentants du gouvernement, du patronat et des syndicats au début avril 1982, le gouvernement étale ses options. Face à des besoins financiers de plus de $700 \$$ millions il n'a le choix que d'abolir certains programmes, mettre à pied quelque 38000 employés ou geler la rémunération des 320000 employés des secteurs public et parapublic. Rejetant les deux premiers, il demande aux syndicats d'accepter le gel proposé ${ }^{38}$. Lors de son discours du budget le 26 mai 1982, le ministre des Finances précise la politique du gouvernement: si les syndicats refusent la demande de surseoir aux augmentations de traitements prévues à la fin des conventions collectives de 1979-1982 pour le $1^{\text {er }}$ juin et le 31 décembre, le gouvernement récupèrera ces montants de manière unilatérale au premier trimestre de 1983.

Les syndicats ayant refusé sa demande, le gouvernement fait adopter le projet de loi 70, le 23 juin 1982, qui lui permet de prolonger par décret les conventions collectives pendant trois mois au début de 1983 et de récupérer ainsi quelque $18 \%$ de leurs traitements.

Après une période de négociations infructueuses à l'automne le gouvernement y met fin en déposant le projet de loi 105 qui fait adopter 109 décrets de plus de 80000 pages au total imposant les conditions de travail de tous les employés des secteurs public et parapublic jusqu'au 31 décembre $1985^{39}$.

La loi 105 et les décrets n'ont pas mis fin au conflit. De nouvelles négociations au début de 1983 sont vite arrivées à l'impasse et le front commun déclenche la grève progressivement pendant la dernière semaine de janvier. Une entente est signée

37. La Presse du 25 novembre 1981 et The Gazette du 2 novembre 1981.

38. Le Devoir et La Presse du 7 avril 1982.

39. Le Devoir les 9, 10 et 13 décembre 1982. 
le 31 janvier avec le SFPQ avant que ses membres ne se mettent en grève. À son tour la Fédération des affaires sociales accepte les propositions le 10 février. Il reste deux groupes de récalcitrants, les professionnels du gouvernement et les enseignants. Sous menace de congédiements, le SPGQ met fin à une grève de huit jours le 8 février, mais promet une campagne de résistance. Quant aux enseignants des niveaux primaire, secondaire et collégial, ils subissent le projet de loi 111 du 16 février, la législation la plus sévère connue au Québec pour mettre fin à leurs grèves illégales (parce qu'interdites par la loi 105). Malgré cette loi, qui suscite la réprobation d'un grand nombre d'observateurs (dont la Commission des droits de la personne et le bâtonnier du Barreau du Québec) les enseignants maintiennent leur résistance jusqu'au 19 avril $^{40}$.

Dans le cas du SPGQ leur opposition au décret prend la forme d'une "opération dénigrement», une campagne de fuites destinées à embarrasser le gouvernement. Cette opération s'attire aussi une critique générale dans les médias, car on considère que les professionnels abusent de leur position de confiance, même si leur cause peut être juste ${ }^{41}$. Quand le décret leur est imposé à la mi-mai ils sont les derniers syndiqués des secteurs public et parapublic à résister encore. La signification de leur lutte réside dans le fait que ce groupe est constitué de jeunes technocrates qui constituent l'élite de la fonction publique depuis la création des corps de professionnels en 1966-1967. Or, après avoir été contesté dans leur rôle de source principale d'informations et de conseils gouvernementaux, après la perte des perspectives d'expansion offertes par le référendum, voici qu'ils sont aux prises avec un contrôle de leurs présences au travail et une sécurité d'emploi régionale réduite à six mois après lesquels ils devront

40. Voir les textes des prises de position dans La Presse du 17 février 1983.

41. Le Devoir des 11 et 12 avril, La Presse le 21 mars et le 13 avril 1983. 
accepter une mutation partout où leurs services pourront être utilisés $^{42}$.

Ainsi, au moment de préparer l'avant-projet de loi sur la fonction publique en juin 1983, le gouvernement du Parti québécois se trouve dans une nouvelle position face à l'administration. Après l'expansion des années 1970, la crise financière sévit et les gouvernements sociaux-démocrates, selon un mot de René Lévesque, doivent devenir bons gestionnaires ou se faire renvoyer par les électeurs ${ }^{43}$. Pour faire face à des pressions qui viennent de l'extérieur de l'État, le gouvernement cherche une bonne partie de la solution à l'intérieur de ses appareils par des mesures visant à diminuer les coûts et à accroître l'efficacité des employés.

\section{L'impatience des députés}

Pendant que les attitudes et la pensée du gouvernement évoluent de la manière décrite précédemment, les députés mènent une réflexion qui aboutit à une remise en question de certaines doctrines constitutionnelles concernant les rapports entre le parlement et l'administration. Les deux approches seront reliées par la création de la Commission Bisaillon en septembre 1981.

C'est le député libéral Claude Forget qui amorce la réflexion avec un rapport intitulé Démocratiser le parlement, préparé en 1977. Ayant été sous-ministre puis ministre des Affaires sociales, M. Forget est bien placé pour apprécier les problèmes des deux côtés de la barrière. Son jugement est sévère: «Le contrôle par-

42. Louis Falardeau, "Comme une profonde blessure... les cinq points majeurs du désaccord", La Presse du 12 février 1983 et Le Devoir du 5 mai 1983. Neuf mois plus tard, le SGPQ considère que cinq dossiers sont toujours en litige, Le Devoir du $1^{\text {er }}$ février 1984.

43. Interview accordée au Devoir le 6 juillet 1981. Le même sort attend le gouvernement socialiste français un peu plus tard, voir J.-M. Colombani, "Gérer la crise, cauchemar de la gauche», Le Monde du 6 avril 1983, reproduit dans Le Devoir du 9 avril 1983. 
lementaire de l'Administration publique est aujourd'hui inexistant ${ }^{44}$.» Le mécanisme inventé au $19^{e}$ siècle pour assurer ce contrôle, la responsabilité ministérielle, a "un caractère fictif ", le ministre étant incapable de suivre tout ce qui se passe au sein de son ministère. Il n'a de choix que d'endosser les actes de ses fonctionnaires ou de les dénoncer pour insubordination.

Le rapport Forget se propose de résoudre ces problèmes en rendant les administrateurs de l'État directement responsables devant les commissions parlementaires des aspects administratifs de leur travail. Réservant au ministre et à ses collègues la détermination et la défense des politiques, le rapport identifie cinq critères selon lesquels les hauts fonctionnaires pourraient être évalués par les députés: i) la fidélité dans l'exécution des politiques, ii) l'exactitude et l'honnêteté, iii) l'économie ou l'efficacité, iv) le respect des droits et libertés personnelles et v) la non-discrimination entre citoyens et citoyennes, employés ou clients.

À cette même époque à Ottawa, il y a une commission d'enquête dont le mandat est similaire au sujet d'étude de Claude Forget et qui a contribué à la diffusion d'un néologisme au Québec. Il s'agit-de la Commission royale d'enquête sur la gestion financière et l'imputabilité (la Commission Lambert) qui a été créée en novembre 1976 après que le Vérificateur général du Canada ait lancé un avertissement à l'effet que le parlement fédéral perdait le contrôle, lui aussi, de l'administration et des dépenses publiques. Dans son rapport de 1979, la commission prend une position proche de celle de Claude Forget. Les sous-ministres, dit-elle, devraient répondre devant le comité des Compres publics de leur gestion et notamment du rendement administratif de leur ministère ${ }^{45}$. Avec ce rapport, le mot imputabilité, version française

44. Claude Forget, "L'administrateur public: sujet ou objet du pouvoir politique", Administration publique du Canada, vol. 21 (1978), 234-242.

45. Rapport de la Commission royale d'enquête sur la gestion financière et l'imputabilité, Ottawa, 1979, 24, 215-217 et 429. Pour une excellente analyse critique, voir Douglas 
de l'anglais «accountability» entre dans le langage de l'administration à Québec comme à Otrawa.

Un peu plus tard à Québec, un autre ancien ministre, Denis Vaugeois, poursuit la réflexion sur la manière de restaurer l'équilibre entre le législatif et l'exécutif au Québec. Il endosse la proposition des rapports Forget et Lambert d'introduire une distinction entre la responsabilité politique et administrative, considérant que les trois grandes contraintes aux réformes parlementaires sont la responsabilité ministérielle, la solidarité ministérielle et la ligne du parti ${ }^{46}$. L'Assemblée nationale a besoin de plus de pouvoirs d'initiative et d'étude, de moyens plus vastes pour contrôler l'exercice du pouvoir règlementaire et les organismes autonomes et, enfin de méthodes qui lui permettront de mieux étudier le budget et de mieux surveiller les dépenses publiques.

Le rapport Vaugeois fait état d'un certain mépris des journalistes pour les députés et leur travail parce que, sur presque toutes les questions, les jeux sont faits lorsque les députés interviennent (les projets de loi, les budgets, etc. étant déjà prêts) et la discipline de parti les empêche de jouer un rôle utile. Depuis une quinzaine d'années les journalistes ont souvent l'impression que les députés font du théâtre pendant que les vraies affaires d'État sont réglées par les ministres et les fonctionnaires ${ }^{47}$.

Dans un tel contexte, on comprend pourquoi les députés ont bien accueilli en novembre 1981 la proposition de la ministre de la fonction publique, Denise Leblanc-Bantey, de créer une commission spéciale composée de députés des deux partis et secondée par une équipe de fonctionnaires, pour étudier la fonction publique et son avenir.

Hartle, «The Report of the Royal Commission on Financial Management and Accountability (The Lambert Report): A Review" dans Canadian Public Policy/Analyse de politiques, vol. 5, 3 (1979), 366-383.

46. Denis Vaugeois, L'Assemblée nationale en devenir, Québec, janvier 1982.

47. Cf. Michel Roy, "Le spectacle continue...", Le Devoir du 27 janvier 1967 et Gilles Provost, "On a laissé filer l'Hydro», ibid. du 9 mars 1981. 


\section{La loi 50 et ses séquelles}

Au moment de la création de la Commission Bisaillon à l'automne de 1981, la Loi sur la fonction publique n'a que trois ans d'existence et déjà les gestionnaires des ministères et organismes se plaignent de la lourdeur et des rigidités introduites par la loi. Pour comprendre le désir d'un changement chez ceux qui s'intéressent le plus directement à cette loi, il faut rappeler brièvement ses origines et son contenu.

À l'origine de la loi 50 de 1978 , il y avait un mouvement qui provenait du Parti québécois et un autre porté par des hautsfonctionnaires depuis quelques années déjà. Le programme du Parti québécois de 1975 avait placé à la tête de la liste des principes de réforme l'idée que:

La nomination de tous les fonctionnaires... se fonde sur un critère unique, celui de la compétence reconnue par des concours publics.

Au printemps de 1975 les dirigeants du parti ont précisé leur pensée en dénonçant les lenteurs dues à la centralisation excessive de la gestion du personnel, l'arbitraire qui règnait lors des promotions, l'absence d'indicateurs précis du rendement des fonctionnaires et le recours abusif à l'engagement d'occasionnels pour faire le travail d'employés permanents ${ }^{48}$.

L'équipe de fonctionnaires chargée de préparer des amendements législatifs a pu se servir des études menées pour le compte du gouvernement Bourrassa vers la fin de son mandat. Leur diagnostic est similaire à celui du Parti québécois, mais il apporte d'autres précisions: tout en constatant l'absence de normes rigoureuses de sélection au mérite et la centralisation excessive qui règne dans la gestion du personnel, les fonctionnaires s'interrogent sur les chevauchements qui existent entre les responsabilités du Conseil du Trésor, du ministère de la Fonction publique et de la

48. Communiqué de presse de René Lévesque et Robert Burns du 28 mai 1975. 
Commission. À la Commission, notamment, on observe un mélange de fonctions de gestion et de surveillance qui fait qu'elle est appelée souvent à décider des appels de ses propres décisions ${ }^{49}$.

L'option choisie renforce à la fois le ministère de la Fonction publique et le régime du mérite. La décision touchant le ministère est surprenante car les différentes études menées sous le gouvernement libéral avaient recommendé son abolition, solution à laquelle s'était rallié le dernier titulaire libéral, Oswald Parent ${ }^{50}$. Il semble que la décision contraire s'inspire de deux motifs: d'une part le nouveau ministre de la Fonction publique, Denis de Belleval, est très intéressé par la réforme et veut y être associé; d'autre part, les fonctionnaires du Conseil du Trésor ne sont pas intéressés à récupérer les fonctions du ministère touchant la négociation des conventions collectives des fonctionnaires ni les autres tâches d'exécution dévolues au ministère ${ }^{51}$.

Le ministère de la Fonction publique devient, par cette réforme, l'unique responsable des politiques générales des ressources humaines au sein de l'appareil étatique. Avec le transfert des questions de classification des emplois, du classement des postes et des conditions de travail du personnel non-syndiqué, il achève la récupération des pouvoirs de gestion de la Commission de la fonction publique commencée avec sa création par la loi de 1969. Désormais il jouit de pouvoirs d'élaboration de règlements, de politiques et de directives dans toutes les facettes de la gestion des ressources humaines à l'exception du recrutement et de la sélection des fonctionnaires. Il continue à être responsable des relations de travail dans la fonction publique et à négocier les conventions collectives des fonctionnaires dans le cadre des mandats

49. André Gélinas, «La conceptualisation d'une réforme” dans Bauer et Cabatoff, Bilan de la loi 50, 91-92.

50. Index cumulatif, La fonction publique du Québec, 1964-1978, Assemblée nationale, $1979,7$.

51. A. Gélinas, loc. cit., 98. 
reçus du Conseil du trésor. Pour la plupart des questions relevant du domaine de la gestion du personnel, le Conseil du trésor agit à la place du Conseil exécutif. C'est lui qui approuvera les règlements prévus par la nouvelle loi ${ }^{52}$.

Sur le plan des structures, un autre grand changement est introduit par la loi 50. Un Office de recrutement et de sélection du personnel de la fonction publique est créé qui hérite des responsabilités de la Commission de la fonction publique en matière de recrutement et de sélection des candidats à la nomination et à la promotion de la fonction publique. Comme c'est le cas pour le ministère, l'Office élabore des règlements qui sont soumis au Conseil du trésor pour approbation. Dépouillée de ses tâches de gestion, la Commission de la fonction publique devient un organisme de conseil et de surveillance chargé d'assurer le respect du principe du mérite. Pour assurer leur indépendance, les membres de la Commission et de l'Office sont nommés par un vote des deux-tiers de l'Assemblée nationale pour un mandat de sept ans.

Le renforcement du principe du mérite est fait par plusieurs dispositions dont les plus importantes se trouvent à l'article 67 de la loi:

Le personnel de la fonction publique est recruté et promu par voie de concours et tout concours doit être de nature à constater impartialement la valeur des candidats.

La sélection est établie sur la base de critères de compétence et d'aptitudes et le concours donne lieu à l'établissement d'une liste classant les candidats par ordre de mérite.

Les nominations et promotions sont faites selon cet ordre parmi les candidats qui ont fait l'objet d'une déclaration d'aptitudes.

Cet article innove par ses précisions quant au type d'épreuve prévu lors d'un concours et surtout par l'exigence de suivre l'ordre

52. Denis de Belleval, "La nouvelle loi sur la fonction publique de 1978" dans Bauer et Cabatoff, op. cit., 109-115 et Jacques Bourgault, «L'organisation de l'administration publique québécoise: 1868-1979" dans l'Annuaire du Québec 1979-1980, 126-132. 
sur la liste d'éligibilité, ce qui n'était pas le cas auparavant. Deux autres articles concourrent à hausser les exigences de qualité chez les fonctionnaires. Pour la première fois, on introduit la possibilité de rétrograder un fonctionnaire pour motif d'incompétence. Aussi, on renverse la pratique existant depuis 1969, qui permet aux membres des cabinets des ministres d'accéder à la fonction publique sans passer par un concours.

Pour éviter de renforcer la concentration des pouvoirs entre les mains des organismes centraux, la loi prévoit la possibilité de déléguer les pouvoirs de l'Office et du ministère en matière de recrutement, de sélection et de classement aux sous-ministres ou aux dirigeants d'organismes qui relèvent de la loi et ceux-ci peuvent les déléguer à leur tour à d'autres agents. Sous réserve des obligations de tenir les concours exigés par la loi, le ministre ou le chef d'un organisme est habilité à faire la nomination, la promotion, la mutation, la rétrogradation ou la destitution des fonctionnaires autres que les sous-ministres et les sous-ministres adjoints et associés.

Les critiques adressées par les syndicats de fonctionnaires et certains observateurs concernaient la politisation de la gestion du personnel et un rétrécissement des droits syndicaux ${ }^{53}$. La première version du projet de loi, la loi 53, fut déposée le 26 juillet 1977 , la veille de la création de la Commission Martin-Bouchard sur les négociations collectives dans les secteurs public er parapublic. Les syndicats de fonctionnaires, qui n'avaient pas été consultés pendant la préparation du projet de loi 53 , ont attaqué celui-ci comme ayant des incidences sur le régime syndical et demandaient la remise de son adoption jusqu'au dépôt du rapport, ce que le ministre a finalement consenti. Malgré quelques concessions faites dans la deuxième version, la loi 50 exclut toujours des matières

53. Voir l'échange entre Alain Baccigalupo et Denis de Belleval dans Le Devoir les 1,2 et 10 décembre 1977 . 
négociables toute question de nomination, de promotion, de classification et de classement des emplois, de permanence, de normes d'éthique et de discipline ainsi que d'organisation (article 116). Le pouvoir du ministère d'agir de façon unilatérale par voie règlementaire inquiète les syndicats.

La politisation est aussi redoutée, en raison de la concentration de pouvoirs au ministère de la Fonction publique et des possibilités de délégation en faveur des ministères et organismes. Les syndicats invoquent le danger d'un retour au patronage. À toutes ces critiques, le gouvernement répond par le rôle d'arbitre et de surveillant attribué à la Commission maintenant libérée de toute responsabilité de gestion et par l'ajout du contrôle des concours de promotion aux tâches de l'Office. Dans la première version de la loi, on avait maintenu le droit d'intégration à la fonction publique de tous les membres de cabinets ministériels après 12 mois de service; sujet, cependant, à l'obtention d'une déclaration d'aptitudes par l'Office. Face à la critique, on a adopté l'exigence de passer par un concours comme tout autre candidat. Finalement, du côté de la politisation, une innovation qui n'a pas soulevé d'objections importantes: désormais, un fonctionnaire pourra démissionner afin de se porter candidat à une élection fédérale ou provinciale et, s'il est élu, il garde le droit d'être réintégré à la fonction publique s'il cesse d'être député avant l'expiration d'un délai de soixante mois après son élection (article 101). Auparavant, il ne pouvait demander d'être réintégré à son poste que s'il perdait son élection. Il est toujours interdit à un membre de la fonction publique de se livrer à un travail de nature partisane pendant une campagne électorale fédérale ou provinciale (d'où la nécessité de l'interprétation donnée à cet article pendant la campagne référendaire de 1980).

Par la loi de 1978, le gouvernement affiche son intention de prendre en main la gestion du personnel, laissant aux organismes autonomes le soin d'assurer le respect du régime de mérite. Tout en cherchant plus de flexibilité par les délégations de pouvoir aux 
ministères et organismes, la loi met en valeur la recherche de l'efficacité par le mandat qu'elle accorde au ministère et par la recherche de la compétence qu'elle exige à toutes les étapes de la carrière d'un fonctionnaire.

Dix-huit mois après l'adoption de la loi, le ministre de Belleval est satisfait des progrès sur le front du régime de mérite, mais il considère que les résultats sont à venir en matière d'efficacité ${ }^{54}$. Le ministère s'affaire à produire des règlements dont près de 130 sur la classification des emplois. Pour comprendre la situation au moment de la Commission Bisaillon et de la réforme de 1983, il sera utile de signaler deux développements concernant la loi, l'un impliquant les cadres supérieurs, l'autre l'égalité dans l'emploi.

Dans la recherche de l'efficacité, le ministère de la Fonction publique est beaucoup moins impliqué que le Conseil du trésor et le vérificateur général, car cette recherche a pris la forme de l'évaluation de la gestion et des programmes ${ }^{55}$. Néanmoins, le ministère introduit en 1978 l'évaluation du travail des cadres supérieurs, élément qui manquait depuis l'introduction du système de notation des autres employés une douzaine d'années plus tôt. Tel qu'amendé en 1979 ce nouveau système lie les hausses de traitement des cadres supérieurs à leur succès dans la rencontre des «attentes signifiées» par les évaluateurs aux cadres supérieurs au début de la période couverte par l'évaluation. Par cette méthode, on espère adapter les exigences aux conditions de chaque poste de cadre et aussi baser l'évaluation sur des faits précis touchant le rendement plutôt que des traits de personnalité. Malgré des faiblesses, cette méthode est aujourd'hui appliquée à la grande majorité des cadres supérieurs ${ }^{56}$. Elle pourrait fournir des éléments

54. Denis de Belleval, "La nouvelle ici...", 116.

55. Alphonse Riverin et Jean Hamel, L'évaluation dans le secteur public québécois, Les cahiers de l'ENAP, $\mathrm{n}^{\circ}$ 5, juillet 1982, 22-30.

56. Marcel Proulx et Michel Roy, L'évaluation du rendement des cadres supérieurs dans la fonction publique québécoise, Québec, ENAP, 1983. 
pour la mise en place d'un système d'imputabilité des hauts fonctionnaires, car elle constitue une expérience de type contractuel.

Les programmes d'égalité en emploi, datent du début des années 1980. En février 1980, le gouvernement adopte une politique visant à redresser la situation des femmes dans la fonction publique. Ce programme est incitatif (comme les précédents) mais il précise de nouvelles structures: un sous-ministre adjoint féminin est chargé du dossier au ministère de la fonction publique, chaque ministère devra désigner un responsable et un comité de surveillance est créé avec des représentants non seulement des organes de gestion du personnel, mais aussi du Secrétariat d'État à la condition féminine, du Conseil du statut de la femme, de la Commission des droits de la personne ainsi que des deux principaux syndicats, le SPGQ et le SFPQ.

En mai 1981, la notion d'égalité en emploi est élargie pour inclure les non francophones et les handicapés. Le projet de loi 12, adopté le 12 juin 1981 charge le ministre de la Fonction publique d'élaborer et proposer des mesures visant à assurer l'égalité en emploi dans la fonction publique, de prévoir les mécanismes nécessaires et de permettre au ministre ou à l'office de regrouper les candidats d'un même niveau et de limiter l'admissibilité aux concours en fonction de bassins de population ${ }^{57}$. Ces amendements reflètent non seulement le désir d'introduire des mesures d'égalité en emploi, ils témoignent aussi des ridigités de la loi de 1978 qui n'avait pas été prévues, comme par exemple, la sélection sur une base régionale. Ces rigidités sont renforcées par la baisse du recrutement, pour l'année 1981-1982 on propose de réduire les effectifs de la fonction publique de $12 \%$ sans recourir aux mises

57. Plusieurs de ces informations proviennent d'un texte inédit de France Nadeau, "Les femmes dans la fonction publique du Québec et le programme d'égalité des chances en emploi », Département de science politique, Université de Montréal, avril 1984. 
à pied, par le non-remplacement des employés qui quittent leur emploi ou qui prennent leur retraite ${ }^{58}$.

La crise financière, donc, rend très difficile la mise en application des programmes d'égalité en emploi. Pour pallier ces faibles résultats, se servant d'une clause de la loi de 1981, la ministre Denise Leblanc-Bantey annonce, au mois de mars 1982 que désormais, on groupera ensemble, comme étant de même niveau, les personnes ayant obtenu les meilleurs scores dans une gamme de dix points sur 300 par rapport à celui du candidat en tête de liste lors d'un concours. Si, parmi les personnes à ce niveau il y a une femme, elle sera choisie pour l'emploi en question ${ }^{59}$. C'est une mesure qui s'écarte de manière importante de la règle du mérite introduite par la loi de 1978 et qui sera reprise dans celle de 1983.

De toute façon, jusqu'en 1983 , les progrès en ces programmes sont décevants. De 1980 à 1983 (au 31 mars) le pourcentage de femmes dans l'ensemble de la fonction publique a progressé de 33 à $35,8 \%$, celui de professionnelles de 15,3 à $17,4 \%$, celui des cadres supérieurs de $2,5 \%$ à $5,65 \%$. Dans ce dernier cas, on pourrait considérer comme une réussite le fait d'avoir plus que doublé les effectifs féminins cadres supérieurs en passant de 71 femmes en 1980 à 190 en 1983, mais la présidente du Conseil du statut de la femme rappelle que si on continue d'augmenter le nombre de femmes cadres supérieurs à un taux d'environ $1 \%$ par année ${ }^{61}$ :

58. Le Devoir du 10 juillet 1981.

59. Journal des débats, Commission permanente de la fonction publique, le 29 septembre 1983 , B-7816.

60. Ministère de la Fonction publique, Présence des bommes et des femmes dans la fonction publique québécoise, vol. 5, 1983, 105-106.

61. Journal des Débats, Commission permanente de la fonction publique le 29 septembre 1983 , B-7816. 
À ce rythme d'augmentation, dans 45 ans, soit en 2028, les femmes auront le privilège de former la moitié de la haute fonction publique québécoise.

Tout en reconnaissant que le gouvernement ne s'est jamais donné pareil objectif, on conçoit que les personnes intéressées trouvent le progrès trop lent.

Du côté des minorités culturelles, il n'y a pas de progrès, mais un recul. Malgré l'existence du nouveau programme, leur présence passe de $2,7 \%$ en 1979 à $2,5 \%$ en $1982^{62}$.

Pour conclure, en 1981 déjà on a dû amender la loi de 1978 pour la rendre plus flexible et pour reconnaître l'importance accordée aux nouveaux programmes d'égalité en emploi. Le recrutement a presque cessé. Par contre, un nouveau système d'évaluation des cadres supérieurs a été introduit avec un certain succès.

\section{La réforme de 1983}

Le processus de réforme qui mène à l'adoption du projet de loi 51 en décembre 1983 a ses origines dans la décision de créer la commission spéciale de l'Assemblée nationale sur la fonction publiqué en novembre 1981. La ministre Denise Leblanc-Bantey a pris la décision de créer la commission, mais c'est cette dernière qui est le catalyseur de la réforme.

\section{Le catalyseur: la Commission Bisaillon}

Mme Leblanc-Bantey est nommée ministre de la Fonction publique à la suite de l'élection du 14 avril 1981. Elle pilote aussitôt les amendements à la loi 50 qui sont adoptés au mois de juin, mais elle ressent le besoin de procéder à une évaluation plus

62. Ministère de la Fonction publique, Les communautés culturelles et la fonction publique du Québer, Inventaire de 1982, Québec, 1983, 11-12. 
en profondeur de cette loi. La solution trouvée à l'automne comble le désir des membres de l'Assemblée nationale de jouer un rôle plus actif: on confiera à une commission spéciale composée de sept députés dont trois de l'opposition, la tâche de faire le bilän de la loi. À cette fin les députés seront secondés par une équipe d'une trentaine de fonctionnaires prêtés par l'Assemblée nationale et par plusieurs ministères.

La Commission procède à de larges consultations en deux temps; au mois de janvier elle rencontre des groupes et individus à huis clos et à la fin d'avril et au début de mai, elle tient des audiences publiques qui permettent aux intéressés de prendre position sur un document de consultation qu'elle a publié au mois de mars. Ces consultations impliquent les représentants d'une quinzaine d'organismes publics, de six syndicats de fonctionnaires, des sous-ministres et des cadres supérieurs, de l'École nationale d'administration publique (ENAP) ainsi que de la Chambre de commerce du Québec. Une quinzaine d'individus, fonctionnaires et professeurs, se sont aussi présentés devant la commission. À sa demande, les effectifs fonctionnaires ont produit pas moins de quarante-huit textes et travaux. C'est tout un exploit que d'avoir réussi à réunir autant de consultations et d'expertises et de déposer son rapport le 11 juin, soit moins de sept mois après sa création.

Les grands thèmes du rapport apparaissent déjà dans son titre: Pour une fonction publique sensible aux besoins des citoyens, moderne, efficace et responsable ${ }^{63}$. En effet, le rapport Bisaillon propose au lecteur trois grands principes: les citoyens ont droit a des services efficaces et de qualité, l'égalité des chances doit avoir la même importance que la règle du mérite dans la gestion de la fonction publique, et les fonctionnaires doivent rendre compte de leur

63. Québec, le 11 juin 1982. Nous nous basons aussi sur une conférence donnée par M. Bisaillon à l'Université de Montréal, Département de science politique, le 6 décembre 1982. 
gestion sur la base de critères tirés des deux premiers principes. L'idée que la fonction publique existe pour bien servir les citoyens peut sembler banale, mais la législation n'a jamais affirmé un tel principe. La tradition amenait le législateur à traiter des droits et obligations des fonctionnaires en termes de leurs rapports avec leurs patrons politiques et administratifs. Jusqu'à présent, il a toujours été du ressort de l'homme politique de définir les besoins des citoyens et une bonne partie des électeurs a vu en leur député un protecteur du citoyen capable d'intercéder en cas de difficulté avec l'administration. Nous verrons plus loin que les implications du revirement proposé ne sont pas encore certaines. En ce qui concerne le mérite, les députés membres de la commission le maintiennent au niveau des grands principes de l'administration publique, mais il n'est pas le seul. Ils notent que déjà, en 1981, pour des raisons d'efficacité et de coût, il a été nécessaire d'assouplir la règle du mérite comme s'appliquant à tout le territoire en tout temps. De plus ils considèrent nécèssaire et possible «de concilier une fonction publique fondée sur le principe du mérite avec une représentation équitable de chaque secteur de la communauté nationale ${ }^{64}$. Une notion clé dans ce rapport est celle de l'imputabilité. Elle est définie de la manière suivante ${ }^{65}$ :

...un mandataire est responsable de la réalisation de son mandat devant son mandant. Par ailleurs, un régime d'imputabilité differe fondamentalement d'un régime de responsabilité parce qu'il requiert la participation objective d'une tierce partie... au plan de l'évaluation de la réalisation des mandats...

Aux yeux de la commission cette tierce partie serait le Vérificateur général qui, sous un mandat élargi, ferait rapport à une commission de l'Assemblée nationale chargée de surveiller l'administration.

La commission rejoint ici les rapports Vaugeois et Forget en proposant de distinguer l'imputabilité politique (donc minis-

64. Rapport Bisaillon, 17.

65. Ibid., 32. 
térielle) et administrative. Celle-ci porterait sur le rendement des fonctionnaires, notion qui serait élargie, cependant, pour tenir compte de l'exigence du service à la clientèle. Pour qu'un tel système puisse fonctionner, aux yeux de la commission, deux grands changements seraient nécessaires. D'abord, il faudrait libérer les gestionnaires des contrôles préalables étouffants provenant des organismes centraux, concrétisés souvent dans les nombreux règlements qu'ils adoptent. L'imputabilité suppose une délégation de pouvoirs assortie de l'obligation de rendre compte de leur utilisation après le fait. Le second changement serait l'introduction d'un système de type contractuel pour les sous-ministres et les cadres supérieurs spécifiant les objectifs qu'ils devraient poursuivre et les indicateurs par lesquels ils seraient jugés par la suite. Ces critères comprendraient l'exigence de mettre en place des mesures pour améliorer le rendement et le service à la clientèle. Si peu de ministères se sont montrés empressés de faire l'évaluation de leurs programmes, c'est qu'il n'y avait aucune incitation à le faire, que ce soit par une punition ou une récompense.

La commission est consciente qu'il faudrait procéder avec soin, faire des expériences et ne pas tout bouleverser dans la fonction publique. Elle suggère deux ordres de priorité: d'une part, on devrait commencer par les organismes autonomes pour ensuite étendre le système aux ministères et aux organismes centraux (p. 45); d'autre part, dans chaque unité soumise à un régime d'imputabilité on devrait commencer par le personnel de direction, pour ensuite inclure les fonctionnaires ayant un contact direct avec le public avant de passer aux strates intermédiaires (p. 28). En tout cas, la clé de tout le système serait l'imputabilité des sous-chefs, c'est-à-dire les sous-ministres et les dirigeants d'organismes, devant l'Assemblée nationale.

La commission porte un jugement sévère sur la gestion des ressources humaines. L'existence d'un groupe de 11400 occasionnels à côté des 60,000 membres de la fonction publique permanente 
et la création de plus de 7000 emplois exemptés de la Loi sur la fonction publique depuis 1976 par la création d'organismes autonomes, sont des signes des rigidités du système auxquelles les gestionnaires tentent d'échapper. Un régime d'imputabilité exige plus de souplesse et plus de pouvoirs délégués aux responsables si l'on veut les tenir responsables de leur direction.

De plus, l'existence de personnes surnuméraires à maints endroits dans la fonction publique exige que l'on adopte des politiques privilégiant la mobilité des fonctionnaires. De la classification aux concours, en passant par les conventions collectives, tout doit être repensé afin d'encourager des transferts de personnes mises en disponibilité ou désireuses de changer de poste. La commission donnerait alors priorité au recrutement interne; elle demande même que soit inscrit dans la loi l'ordre de priorité dans les étapes à parcourir pour combler un poste vacant (recours au personnel mis en disponibilité, mutation, promotion ou, enfin, recrurement externe (p. 92 à 95). Pour assurer un certain renouveau, et pour ne pas négliger les droits des jeunes, la commission propose de réserver un pourcentage (non précisé) d'emplois pour le recrutement annuel de finissants de collèges et d'universités.

Il y a bien d'autres points dans ce rapport, mais ce sont les éléments essentiels à notre propos. L'influence du rapport tient à la justesse de son diagnostic aux yeux des autorités, ce qui témoigne, croyons-nous, non seulement de l'esprit non partisan qui a présidé à ses travaux, mais aussi de la qualité de ses recherches et des consultations. Cette interprétation se base non seulement sur l'accueil donné au rapport, mais aussi sur le fait qu'il est loin d'être une étude détaillée. Ses 134 pages dactylographiées renferment pas moins de 139 recommandations, de sorte que plusieurs sont faites sans que le lecteur sache trop quelles en sont les justifications. Il semble bien que ce soit le diagnostic et l'économie générale du rapport qui assurent son impact. 


\section{La proposition gouvernementale}

Le personnel politique et administratif passe l'hiver de 19821983 à préparer une nouvelle loi sur la fonction publique dont il y a, paraît-il, treize versions successives. Au mois de juin 1983, un Avant-projet de loi sur la fonction publique est déposé et l'invitation est lancée aux intéressés de faire leurs commentaires. Il y a trois jours d'audiences portant sur l'Avant-projet à la fin de septembre 1983 et la version définitive du projet est adoptée en décembre.

À l'automne de 1982, il y a plusieurs raisons qui font croire que le rapport Bisaillon rencontre dans ses grandes lignes l'approbation du gouvernement. Nous avons vu que l'un des axes de sa stratégie face à la crise financière était l'idée de réaliser des économies importantes dans la fonction publique sans couper les services. Ceci d'ailleurs devait correspondre à sa lecture des sondages, qui montrent que la majorité des Québécois est favorable au gel des salaires et contre la grève dans le secteur public, mais qu'elle est contre aussi, à $80 \%$, des coupures dans les services de santé ou de l'éducation ${ }^{66}$.

Que les solutions proposées par le rapport Bisaillon soient partagées par le gouvernement est indiqué dans une conférence donnée par Louis Bernard, le 12 octobre 1982, sur le thème "Pour une fonction publique sensible aux besoins du public, compétente et efficace: la voie de l'imputabilité» ${ }^{67}$. À cette occasion, s'adressant à un groupe de fonctionnaires, il note que son titre est tiré presque mot pour mot du mémoire présenté par les sousministres à la commission Bisaillon. Autre signe que la pensée du gouvernement évolue dans ce sens: au moment de supprimer

66. Marc Laurendeau, “Préoccupations économiques: l'écart s'est creusé entre les Québécois et leurs gouvernants», La Presse du 31 mars 1982. Sur le droit de grève, voir aussi La Presse du 4 septembre 1979.

67. Conférence donnée devant la section québécoise de l'Institut d'administration publique du Canada, le 12 octobre 1982. 
les ministères d'État en septembre 1982, le Premier ministre confie à Denis Lazure un nouveau poste rattaché au Conseil exécutif de ministre délégué aux relations avec les citoyens.

C'est donc sous la double consigne d'accroître la productivité et de mieux servir les citoyens qu'est préparé l'Avant-projet de loi sur la fonction publique. Il subira quelques amendements mineurs après les audiences de la Commission permanente de la fonction publique de l'Assemblée nationale en septembre et des rencontres à huis clos de la ministre et des syndicats de fonctionnaires. Devenu le Projet de loi 51, il sera adopté le 21 décembre. Nous le présentons dans ses grandes lignes, quitte à expliciter quelques détails en examinant les enjeux de la nouvelle loi.

La loi 51 introduit cinq changements importants dans la loi de la fonction publique. D'abord, pour la première fois, la loi débute par une déclaration d'objectifs et de principes pour la gestion de la fonction publique. Ensuite, elle réaménage les structures par l'abolition, notamment, du ministère de la Fonction publique. Troisièmement, elle amende la notion de la sélection au mérite par l'introduction de la notion de niveaux. Quatrièmement, elle crée un nouveau corps d'administrateurs d'État. Enfin, elle change les règles d'éthique et de discipline sur les plans professionnel et politique.

En présentant son projet de loi, Mme Leblanc-Bantey indique qu'il vise à rajuster l'administration aux changements survenus dans la société québécoise. Ceci suggère une approche écologique ou contingente, jugement que confirme plus tard l'approche du président du Conseil du trésor. Par contre, quand la ministre précise sa pensée, elle passe immédiatement à un diagnostic interne: pour sévir contre le favoritisme des temps passés, on serait passé à l'autre extrême, "l'abus discrétionnaire a cédé le pas à l'abus

68. Journal des débats, Commission permanente de la fonction publique, le 27 septembre 1983, B-7645-7646. 
réglementaire ${ }^{68}$. Cette approche a donné, dit-elle, une fonction publique fortement centralisée, et bureaucratisée qui, malgré des qualités professionnelles indéniables a fini par «ne fonctionner que pour elle-même».

La réponse du gouvernement se trouve dans une série de principes définis au début de la loi, dont le plus important se trouve à l'article 2 :

La fonction publique a pour mission de fournir au public les services de qualité auxquels il a droit, de mettre en cuvre les politiques établies par l'autorité constituée et d'assurer la réalisation des autres objectifs de l'État.

La ministre insiste sur la primauté de l'objectif service aux citoyens qui vient s'ajouter à celui de mettre en application les décisions de l'Assemblée nationale et du gouvernement ${ }^{69}$.

L'article 3 de la loi indique que la gestion des ressources humaines doit favoriser: 1) l'efficience de l'administration, 2) «un régime selon lequel le fonctionnaire investi... (des) ...pouvoirs de gestion doit en rendre compte selon les moyens mis à sa disposition», 3) l'égalité d'accès de tous les citoyens, 4) l'impartialité et l'équité des décisions affectant les fonctionnaires, et 5) «la contribution optimale, au sein de la fonction publique, des diverses composantes de la société québécoise».

Le principal changement des structures est la disparition du ministère de la Fonction publique. Ses fonctions de responsable de la gestion du personnel vont au Conseil du trésor pour ce qui est des politiques de classification, de dotation et de règles d'éthique et de discipline ainsi que la négociation, la signature, la mise en application et l'interprétation des conventions collectives avec les syndicats de fonctionnaires (chapitre V). Les autres fonctions du ministère de conseiller du gouvernement en matière de gestion des ressources humaines, de rendement, d'organisation et de per-

69. Ibid., B-7647. 
fectionnement et d'organisme commun de formation, de placement de fonctionnaires en disponibilité et de système intégré d'information à la gestion passent à l'Office des ressources humaines, qui succède à l'Office de recrutement et de sélection. Des délégations considérables de pouvoir sont prévues de la part de l'Office aux sousministres et aux dirigeants d'organismes qui sont désormais désignés comme responsables de la gestion des ressources humaines de leur organisation (article 37). Ils nomment les fonctionnaires, tandis que sous l'ancienne loi les ministres les nommaient ou bien leur déléguaient ce pouvoir. La responsabilité du sous-ministre est donc plus nettement dégagée. Celui-ci peut, s’il le juge approprié, déléguer ces responsabilités à ses adjoints ou aux cadres supérieurs (article 38).

Le principe du mérite, l'un des piliers de la loi de 1978, n'est plus mentionné dans la loi 51 , seulement le principe du recrutement et de la promotion par concours (article 40). Plusieurs articles indiquent comment le gouvernement entend limiter l'application stricte du principe du mérite, qui signifiait dans l'ancienne loi, la sélection des candidats strictement selon l'ordre de classement à l'issue du concours. On généralise maintenant la sélection au choix parmi les personnes qui se classent au même niveau lors d'un concours (article 51). L'article 41 dit que lorsque l'Office des ressources humaines fixe les conditions d'admission à un concours il doit respecter les politiques établies par le Conseil du trésor touchant les programmes d'accès à l'égalité et prévoyant le recrutement de personnes employées dans les réseaux de l'éducation ou des affaires sociales. On ouvre la porte ici à la mobilité intersectorielle préconisée par le rapport Bisaillon. D'autre part, la crise financière et le gel du recrutement sont reflétés dans l'article 97, qui permet à l'Office d'adopter des mesures visant à restreindre le nombre de candidatures à un concours.

Le quatrième changement retenu est la création d'un corps d'administrateurs d'État qui comprend les sous-ministres, les sous- 
ministres adjoints ou associés, le secrétaire général du gouvernement et ses associés et le président et le vice-président de l'Office. Cette catégorie est franchement politique: ses membres sont nommés par le gouvernement sur proposition du Premier ministre. Ce qui est nouveau c'est leur intégration dans un corps et la possibilité de les engager à contrat. Étant donné les rôles identiques que l'on attribue aux sous-ministres et aux dirigeants d'organismes, nous ne comprenons pas pourquoi on a omis ceux-ci du corps nouveau, d'autant plus que la mobilité semblait être l'un des objectifs visés.

La dernière catégorie de changements introduite par la loi 51 touche aux conditions professionnelles et politiques qui sont faites aux fonctionnaires. Le régime syndical est reconduit tel quel en attendant le rapport d'un groupe de travail créé par le comité des priorités du Conseil exécutif sur tout le régime des négociations dans les secteurs public et parapublic. Le code d'éthique et de discipline est introduit dans la loi même pour la première fois. La clause qui a retenu le plus l'attention des fonctionnaires est celle de l'article 6 qui dit:

Sous réserve des dispositions relatives à l'accès à l'information et à la protection des renseignements personnels, le fonctionnaire est tenu à la discrétion sur ce dont il a connaissance dans l'exercice de ses fonctions.

Par ailleurs, les droits politiques des fonctionnaires sont clarifiés. Ils doivent faire preuve de neutralité politique dans l'exercice de leurs fonctions et de réserve dans la manifestation de leurs opinions (article 10 et 11 ) mais la vieille distinction entre un travail de partisan pendant ou en dehors des périodes électorales a disparu. L'aspect le plus controversé de la nouvelle loi vient du fait qu'elle limite aux élections provinciales le droit des fonctionnaires de se présenter aux élections et de siéger au parlement (article 23). Par ailleurs, la limite de soixante mois est enlevée, de sorte qu'un fonctionnaire qui cesse d'être député peut en tout temps demander à l'Office des ressources humaines de procéder à une nouvelle 
vérification de ses aptitudes en vue d'un nouveau placement. Le même droit est accordé aux fonctionnaires après une période d'emploi dans un cabinet ministériel ${ }^{70}$. Quand on lui demande pourquoi la loi n'accorde plus les mêmes droits aux fonctionnaires désireux de se présenter aux élections fédérales, la ministre répond simplement que c'est un privilège qui est accordé parce que c'est seulement dans le contexte des lois et politiques québécoises que l'obligation de neutralité s'impose ${ }^{71}$. Néanmoins, la conséquence de cette interprétation sera que le fonctionnaire québécois a le droit de se présenter aux élections provinciales avec droit de réintégration le jour où il sera battu, tandis qu'il lui faudrait demander un congé sans solde, ce qui n'est pas accordé automatiquement, et il n'aura pas de droit précis à la réintégration s'il siège à la Chambre des communes un certain nombre d'années. La même çhose s'applique d'ailleurs aux charges publiques municipales.

\section{Enjeux et prises de position}

Les audiences de la Commission parlementaire sur l'Avantprojet ont permis de prendre connaissance des positions des groupes et intervenants les plus intéressés par la réforme du régime de la fonction publique. Ils se classent en trois grandes catégories: d'abord les regroupements de fonctionnaires, en second lieu certains groupes d'intérêt ou porte-parole comme le Conseil du statut de la femme, l'Alliance-Québec et la Centrale des enseignants du Québec, et troisièmement, un acteur spécialisé, l'École nationale d'administration publique. Il est intéressant de remarquer que les regroupements de fonctionnaires comprennent non seulement

70. Articles 26 et 27 . Ce privilège n'est pas offert aux membres nommés aux cabinets de l'extérieur de la fonction publique.

71. Débats de la commission parlementaire le 27 septembre 1983, B-7660. Outre le député libéral Doyon qui est contre cette restriction, le SFPQ et l'Association des conseillers en gestion préconisent un traitement égal devant toutes les charges publiques. 
les syndicats (le SFPQ et le SGPQ) mais aussi le personnel d'encadrement: l'Association des cadres supérieurs, le Syndicat des cadres (intermédiaires) et le Syndicat des conseillers en gestion du personnel ${ }^{72}$. À ce groupe d'intervenants, on peut ajouter les sous-ministres qui, pour la première fois, ont pris position collectivement et publiquement sur les grands thèmes de cette législation lors de la présentation d'un mémoire à la Commission Bisaillon $^{73}$. Évidemment, le débat sur cette législation est mené par les députés de l'Opposition libérale ainsi que le député indépendant et ancien président de la Commission spéciale, Guy Bisaillon.

De ces interventions un nombre d'interrogations sur les enjeux et le sort probable de cette loi émergent. En général, on peut dire que les résultats de cette loi ne sont pas plus faciles à prévoir que ceux de la loi de 1978. Presque tous les intervenants notent que la nécessité de cette loi prouve l'échec de celle de 1978. L'ENAP et l'Association des cadres supérieurs s'inquiètent des effets déstabilisateurs de changements trop fréquents au cadre législatif. Les cadres supérieurs considèrent qu'il y a eu trop de réformes mal pensées dans le passé et que le temps est venu d'une enquête approfondie qui pourrait dégager une philosophie et un plan d'ensemble. L'opposition note que la plupart des valeurs mises en évidence en cetre occasion ont justifié les réformes de 1969, de 1970 ou de 1978; elle se demande quelles sont les raisons de croire qu'on fera mieux cette fois. Avec l'opposition, M. Bisaillon note qu'on a fait une sélection parmi les recommandations de sa commission et que cette loi ne sera qu'un vou pieux sans d'autres législations pour concrétiser un régime d'imputabilité.

72. Les témoignages se trouvent aux Débats de la commission parlementaire les 27,28 et 29 septembre 1983, pages B-7645 à B-7846.

73. Mémoire des sous-ministres du Gouvernement du Québec à la Commission spéciale de l'Assemblée nationale sur la fonction publique, juin 1982. 
Les deux syndicats de fonctionnaires considèrent que le gouvernement s'est servi des recommandations du rapport Bisaillon pour accrôtre les droits de gérance et ainsi politiser davantage la gestion de la fonction publique. Pour appuyer ces affirmations ils invoquent l'absence de toute définition précise des termes de base; même la fonction publique est définie de manière tautologique car l'article 1 dit que la loi s'applique aux personnes nommées suivant... la loi. On note que les sous-ministres adjoints relèvent du choix du gouvernement, que les sous-ministres auront le choix libre aux concours parmi les candidats d'un même niveau, que l'Office des ressources humaines relève désormais du gouvernement et non de l'Assemblée nationale. On se demande si le fait d'agir par directive plutôt que par règlement rendra l'intervention des organismes centraux moins oppressive pour les gestionnaires des ministères et organismes. Par leur opposition, les syndicats ont réussi à faire enlever de l'Avant-projet certains articles ouvrant la porte à l'arbitraire, notamment celui qui permet au sous-ministre de congédier un fonctionnaire "pour cause juste et suffisante» et un autre qui lui permet d'ajouter des conditions additionnelles de recrutement lors d'un concours.

Les syndicats reprochent au gouvernement d'avoir une fois de plus chambardé la loi de la fonction publique sans toucher au régime syndical, sous prétexte qu'il est à l'étude. Le SGPQ et la CEQ reprochent au gouvernement de ne pas avoir accordé aux fonctionnaires le régime de relations de travail prévu par le Code $d u$ travail, gardant plutôt un régime d'exception sur les plans de l'accréditation, des sujets négociables et des services essentiels à maintenir en cas de grève. Le SPGQ rappelle que le régime d'exception était justifié en 1965 par les pouvoirs exceptionnels qu'on voulait réserver à un organisme indépendant du gouvernement, la Commission de la fonction publique. Puisque la loi 51 achève la transformation de ce système en une administration ordinaire contrôlée par le gouvernement et surveillée par la 
Commission, la raison d'être du régime d'exception a disparu. Guy Bisaillon exprime aussi sa déception car son rapport avait recommandé un rapprochement entre la Loi sur la fonction publique et le Code du travail, mais la ministre n'a rien cédé sur ce point.

En ce qui concerne les grands principes énoncés dans la loi, les intervenants les acceptent, mais avec quelques réserves. Le SGPQ et le Conseil du statut de la femme demandent un engagement plus ferme dans la loi quant à l'accès à l'égalité. Les conseillers en gestion du personnel notent la possibilité de conflits entre l'obligation de servir l'intérêt public et celle de la discrétion, entre l'exigence de l'efficience et celle de l'équité qui, elle, implique le droit d'appel des décisions administratives devant la Commission de la fonction publique. Le SFPQ et Alliance-Québec ont souligné l'ambiguité du principe «fournir au public les services de qualité auxquels il a droit», l'un insistant sur les effets des baisses d'effectifs, l'autre sur la question de la langue de service.

Les cadres supérieurs et intermédiaires sont favorables à l'imputabilité à condition que celle-ci s'accompagne d'une délégation de pouvoirs. Ils endossent le point de vue des sous-ministres qui disaient dans leur mémoire à la Commission Bisaillon ${ }^{74}$ :

...une personne ne peut être imputable à une autre que si elle en a reçu une délégation de pouvoirs et si elle possède une autorité claire et ultime de poser les gestes pour lesquels elle doit rendre des comptes.

Tous ces commentaires ne font que souligner l'incertitude dans laquelle on entreprendra la mise en application de la loi. Les députés ont fait remarquer que la réforme amorcée sera incomplète sans d'autres législations touchant les principes de l'imputabilité, les tâches du Vérificateur général et le régime syndical.

Plus que des changements juridiques à venir, la loi soulève la question de la possibilité de faire changer les mentalités. Madame Leblanc-Bantey a admis lors du débat de la deuxième lecture qu'il

74. Ibid., 7. 
faudrait changer les mentalités au Conseil du trésor, au sein de la fonction publique et parmi les députés de l'Assemblée nationale pour que la réforme puisse se réaliser ${ }^{75}$. Or, sur chaque plan, il y a des doutes sérieux. D'abord, plusieurs intervenants se sont demandés si le Conseil du trésor est l'endroit indiqué pour loger la responsabilité suprême en matière de gestion du personnel à l'heure du service au public et de l'imputabilité. Comme pour confirmer leurs craintes, le président du Conseil du trésor, Yves Bérubé, a donné une version différente de la réforme, lors de son intervention sur le principe du projet de loi. Notre objectif, ditil, est de maintenir des services de qualité au plus bas coût, voire réduire les coûts tout en offrant des services de qualité. Il se félicite que, «notre administration publique a réussi à réduire les coûts des services publics au Québec de tout près de $12 \%$ en l'espace de trois ans», ce qui représente un accroissement de productivité exceptionnel ${ }^{76}$. Le député qui l'a suivi dans ce débat, Reed Scowen n'a pas manqué de souligner l'approche presque exclusivement financière de celui qui devait mettre la loi en application $^{77}$.

Du côté de la fonction publique, chaque groupe intervenant note que son concours sera nécessaire pour bien réussir la réforme. Les syndicats sont particulièrement insistants à cet égard, notant qu'une fois de plus ils n'ont pas été associés à la préparation du projet de loi, ce à quoi la ministre répond que d'une part, le climat des relations de travail n'était pas favorable pendant l'hiver et le printemps de 1983 et, d'autre part, aux yeux des syndicats, la consultation n'est réussie que si elle accepte toutes leurs demandes ${ }^{78}$. Néanmoins, bon nombre d'intervenants s'interrogent

75. Débats du $1^{\text {er }}$ décembre $1983,3646-3649$.

76. Ibid., 3674 .

77. Ibid., 3676-3677. M. Bérubé a été remplacé au poste de Président du Conseil du Trésor par Michel Clair le 5 mars 1984.

78. Débats de la commission parlementaire du 27 septembre 1983, B-7709. 
sur une réforme qui exige la collaboration des fonctionnaires à la lumière des stratégies du gouvernement visant à démontrer que les fonctionnaires sont des privilégiés qui ne travaillent pas assez et qui ne se soucient que de leurs conditions de travail et à la suite des lois 105 et 111 . Les syndicats disent avec plus de force ce que les sous-ministres ont écrit à la Commission Bisaillon ${ }^{79}$ :

La tâche du fonctionnaire a malheureusement été dépréciée et il importe de corriger cette situation car il serait impossible de réaliser une fonction publique sensible aux besoins des citoyens, compétente et efficace si ses employés se sentent dévalorisés et ceci aux yeux même de leurs employeurs et clients, le public.

C'est un défi considérable auquel les débats parlementaires n'ont apporté aucune réponse. Malgré certaines demandes qui lui ont été faites, le gouvernement n’a pas créé de mécanisme ou lieu de consultation permanente avec les syndicats, ni de surveillance conjointe de la mise en application de la loi.

La stratégie du gouvernement mise davantage sur la collaboration des cadres supérieurs. Ceux-ci auront aussi à changer leurs attitudes et comportements. Dans le passé, sans négliger les questions du rendement, leurs soucis principaux touchaient des questions de statut, de carrière et de contrôles centraux ${ }^{80}$. Une étude récente a montré que la grande majorité des cadres supérieurs se préoccupe autant sinon davantage des relations humaines que de la productivité ${ }^{81}$. L'association des cadres supérieurs a compris et accepté qu'un régime d'imputabilité implique que l'on s'intéresse davantage aux résultats. Sans doute l'expérience des «attentes signifiées» sera utile à cet égard, mais il y a des changements à faire ici comme ailleurs.

79. Le Mémoire des sous-ministres à la commission Bisaillon, 11.

80. Rapport du comité d'étude sur la gestion des cadres (rapport Francoeur-Hendriks), Québec, vol. 1, 1975, 34-50.

81. André Leblanc et Luc Poirier, Les cadres de la fonction publique québécoise: styles de leadership, besoins et satisfaction, Québec, ministère de la Fonction publique, 1982. 
On doit aussi soulever des questions sur la possibilité pour le personnel politique de faire la part des choses, de distinguer entre les comptes politiques que l'on pourra demander au ministre et l'imputabilité administrative. Guy Bisaillon reconnaît que les cadres administratifs seront appelés à prendre des décisions de nature politique quand ils auront à traiter de la satisfaction de la clientèle, des droits des clients et des fonctionnaires, de l'accès à l'information, etc. C'est une autre question de savoir si les députés sauront et pourront s'abstenir de tirer des avantages partisans des échanges avec les fonctionnaires imputables. L'expérience des audiences de la commission parlementaire de l'énergie et des ressources sur le comportement des administrateurs de la Société d'énergie de la Baie James dans le règlement à l'amiable avec les syndicats à la suite du saccage du chantier LG-2 en 1974, n'est pas de bon augure ${ }^{82}$. Par ailleurs, le même incident soulève toute la question des attitudes des membres des cabinets politiques face à l'imputabilité et à la délégation de pouvoirs qu'elle implique.

\section{Conclusion}

La réforme de la fonction publique instituée par la loi 51 a trois causes principales. Le gouvernement du Parti québécois a été amené par la crise financière des années 1980 à chercher à accroître la productivité et à comprimer les dépenses de la fonction publique. Les députés se montrent de plus en plus insatisfaits de leur position vis-à-vis du tandem gouvernement-administration, ils remettent en question la doctrine de la responsabilité ministérielle et s'intéressent à la notion de l'imputabilité des fonctionnaires. Au sein de la fonction publique, l'expérience des rigidités issues de la loi 50 de 1978 et les pressions inhérentes à l'introduction

82. Gilles Lesage, "Pénible expérience du procès parlementaire", Le Devoir les 16 et 17 juin 1983 . 
des programmes d'égalité en emploi appellent des changements. Le catalyseur a été la Commission spéciale de l'Assemblée nationale qui, dans un laps de temps très bref, produit un rapport qui attire l'adhésion de principe de la plupart des intervenants. Cependant, la loi telle qu'adoptée laisse planer plusieurs doutes quant à sa réalisation.

En termes de problématiques de réforme, le gouvernement répond à des pressions externes et essaie d'adapter l'administration aux changements survenus dans l'environnement. Il semble compter sur les appuis des députés et des cadres supérieurs qui bénéficieront de la mise en application de la loi. Par contre, les syndicats, de même que les autres intervenants, s'inspirent d'une problématique interne, dans le genre du développement organisationnel, pour poser le dilemme d'une réforme qu'on voudrait faire sans l'adhésion des fonctionnaires.

À un niveau d'observation, donc, l'expérience de cette loi nous dira si une réforme conçue pour répondre à des pressions externes peut réussir contre des résistances internes, autrement dit, si le gouvernement pourra imposer le changement des mentalités que la loi implique.

Cependant, un regard plus attentif indique combien il est difficile d'appliquer à une administration entière les leçons qui proviennent d'expériences menées au niveau micro-administratif, dans des cellules de base. Une réforme qui vise à introduire un changement technologique ou à offrir un meilleur service à la clientèle d'un bureau donné se prête beaucoup mieux à la formulation d'hypothèses simples qu'un événement comme la réforme de la loi de la fonction publique. Dans ce dernier cas, les acteurs sont trop nombreux et les enjeux trop complexes pour en faire un bilan simple.

Repassons en revue nos quatre conditions d'une réforme. réussie. D'abord, il y a la volonté de changement. Celle-ci existe clairement dans le cas présent, mais il faudrait parler des volontés 
de changement. D'une part, le gouvernement, acteur principal, n'a pas des objectifs identiques à ceux de ses alliés, les députés et les cadres supérieurs. D'autre part, le gouvernement n'est pas un acteur ayant des objectifs cohérents et une stratégie unique. Ce qui semble être une réforme d'envergure pour Denise LeblancBantey se présente davantage comme un moyen d'économiser pour Yves Bérubé. Cette incertitude se manifeste dans la loi elle-même dont les nouveaux principes ne sont pas tous réalisables en même temps. Il peut y avoir une opposition entre l'obligation de bien servir le public et celle d'obéir à l'autorité constituée, entre l'exigence de la rentabilité et le respect de toute une série d'obligations concernant l'égalité en emploi, la non discrimination, les droits de la personne et l'accès à l'information.

Ensuite, il y a le catalyseur. Dans le cas présent, nous l'avons identifié comme étant la Commission Bisaillon, mais il y avait d'autres agents propulseurs comme la ministre Leblanc-Bantey ou le Secrétaire général, Louis Bernard. Nous avons noté aussi qu'il y aura rupture entre la conception de cette réforme et son exécution. Celui qui doit assurer la mise en application de la loi (le Conseil du Trésor) n'en est pas l'auteur.

Vis-à-vis de nos deux autres conditions touchant les pressions externes et les appuis internes, notre récit suggère qu'un tel langage erre en supposant une distinction trop nette entre l'administration et le public. L'administration est aujourd'hui une institution au sens sociologique du terme, avec des racines multiples et profondes dans le milieu. Ses différents services ont des liens étroits avec la majorité des secteurs de la société québécoise, de sorte qu'au delà de l'opinion du grand public révélée par les sondages, il y a celle des groupes qui font affaire avec l'administration. Ces groupes peuvent défendre les intérêts de secteurs entiers de la société (dans notre cas ceux des femmes, des minorités culturelles et des syndiqués) ou bien ce sont des intérêts plus spécifiques liés aux activités de ministères et organismes sectoriels. 
Souvent on verra des groupes des deux sortes se concerter avec une administration afin de résister à des coupures budgétaires qui diminueraient les services offerts ou le nombre de postes à combler ou encore l'importance politique accordée à une question. Ainsi l'administration et ses clientèles forment un ensemble bien trop complexe pour être représenté par deux blocs qu'on imagine en conflit ou en parfaite harmonie.

Le temps nous dira si cette réforme aura des effets profonds. Certes, après une période de transformations majeures pendant les années 1963-1968, les réformes depuis 1969 n'ont pas eu les effets escomptés. À cet égard, notre expérience récente est comparable à celle des États-Unis où, selon une étude de March et Olson:

... à long terme, le développement des institutions politiques est moins le produit d'intentions, de plans et de décisions conséquentes que d'adaptations graduelles à des problèmes changeant avec les solutions disponibles et ce, dans les cadres signifiants qui, eux aussi, évoluent ${ }^{83}$.

Mais dans cette évolution, les réformes ne sont pas sans importance. Leurs auteurs contribuent à l'évolution des consciences et ils orientent l'administration vers des solutions qui, si elles sont proposées et soutenues avec persistence, finissent par avoir leur effet. Il s'agirait, selon March et Olson, moins d'une question de génie social que de culture ou de jardinage.

Dans cette perspective, la nouvelle loi de la fonction publique marque l'éclipse d'une rhétorique, celle du mérite, et le couronnement d'une autre, celle du rendement et de l'économie. Cependant, la nouvelle loi devra soutenir la compétition de deux autres discours, celui de l'égalité et celui de la responsabilité politique. C'est cette jonction de valeurs économiques et politiques

83. James G. March et Johan P. Olson, «Organizing Political Life: What Administrative Reorganization tells us about Government», American Political Science Review, vol. 77, no. 2, (juin 1983), 292 (traduction libre). 
qui fait la spécificité de l'administration publique. Les administrateurs qui ont connu les deux disent que l'administration publique est «infiniment plus complexe» que l'administration privée» ${ }^{84}$. Aucune recherche de solutions claires, nettes et définitives ne pourra changer cette donnée fondamentale.

84. L'expression est de Harry Rogers, alors contrôleur général des finances, Ottawa, cité par Joan Cohen, "Ottawa's Managerial Revolution", Report, juin 1979, 12 B. Voir aussi J. W. Macy dans le symposium cité à la note 2, 315 et D. Hartle, loc. cit.. 